\title{
SEMANTIC NETWORK AND CONCEPT MAPPING IN XHY ITEMS
}

\author{
Xingfu Wang ${ }^{1}$ and Guohui Liu $^{2}$ \\ 1. School of Computer Science, Chongqing University, 400030, Chongqing, China. \\ 2. Institute for Languages, Cognition and Information Processing, Chongqing University, \\ 400030, Chongqing, China
}

\begin{abstract}
Semantic network is a popular knowledge representation. The authors in this article apply it to analyze the literary language, xiehouyu(riddle-pun in English) --a unique feature in Chinese literature and daily communication, for the purpose of seeking the activation and mapping of riddle's function to the true meaning of this genre. The key concept in the riddle in xiehouyu items is wellknown information, and generally it has the similarity to the other concept in the riddle therefore forming the dual relationship of mapping between them, thus the simile of this specific langue is formed by comparing the similarities of the two concepts and the metaphor of it is formed by extending the literal meaning of the answer.
\end{abstract}

Key words: semantic network; xiehouyu; concept mapping; metaphor; spreading activation

The application of Artificial Intelligence(AI) in Natural Language Processing(NLP) is brilliant and outstanding, and semantic network becomes a popular knowledge representation ${ }^{[1]}$. Semantic network is the idea of arranging concepts into a hierarchical taxonomy and using this for semantic processing. It is well established in some consultation systems to distinguish animals or mineral cores, for instance, the attempts to represent the knowledge and reasoning processes of experts in the geological domain; and literary concepts are rarely represented in this way. The authors try to describe the spreading activation of semantic network in xiehouyu items with a series of examples containing the word "monk" in the riddle and expects 
to deal with this specific genre with metaphor by depicting the complex relationships.

\section{SEMANTIC NETWORK AND XIEHOUYU}

Semantic network was first introduced by Quillian ${ }^{[2]}$ based on the socalled "associative memory model": the notion that human memory is organized on the basis of association, that humans represent the real-world through a series of associations. In comprehending the transmitted information the hearer depends on his searching and choosing a suitable knowledge network in his memory ${ }^{[3]}$, thus establishing an optimal relation with what has been said. More precisely a semantic network "is defined as a type of knowledge representation that formalises objects and values as nodes and connects the nodes with arcs or links that indicate the relationships between the various nodes: A data structure for representing declarative knowledge. It can be argued that the nodes can also represent concepts, and the arcs the relations between concepts, thereby forming semantic networks" ${ }^{\prime[4]}$.

Minsky's Frames ${ }^{[5]}$ sought to provide the default information for transforming an image of a scene into a linguistic description. Though semantic networks sought to provide a similar foundation for natural language understanding. Its primary motivation was the economy principle stating that items are stored without redundancy and could be realized in computer environments on reasoning.

Chinese culture has a long history and its literature works contain unique accumulated features. In the development of Chinese culture some genres such as idioms, proverbs, and xiehouyu items were created. In this article the authors prefer the spelling of xiehouyu because its unique features in Chinese even though some scholars used "rest-ending sayings", "post-pause expressions", "example-explanation sayings", "enigmatic folk similes"[6], "two-part allegorical sayings" or "riddle-puns" "[7]. And the authors put xiehouyu for short in the title of this article. It's hard for people to decompose a text into single elements in hopes of finding equivalents in the target language. We should keep in mind that the content expressed grammatically in one language may be expressed lexically in another language. If no grammatical forms are available in the target language, the translator must look for lexical means to render the same semantic content. Therefore the translation here is not of vital importance, the purpose is to demonstrate this genre in English. The similarities in two languages would permit the semantic equivalence translation but the difference in them would create the unique genres in them. 
Xiehouyu is a unique Chinese fixed expression and is a popular form with common people. Xiehouyu is defined as "a two-part allegorical saying, of which the first part, always stated, describes a riddle, while the second part, sometimes unstated, carries a message as an answer to the riddle. ${ }^{,[8]}$ The form of this genre in the xiehouyu dictionaries is usually linked with a dash, with the first part usually being a metaphor and the second part mainly the further explanation to the first part, and the dash being equal to the predicators "is" or "are". In this article the first and second parts are called the riddle and the answer. The explanatory function of xiehouyu requires the allegorical part being a well-known and outstanding concept ${ }^{[9]}$. There are 37 xiehouyu items containing the word 'monk', and this outnumbers those items containing 'Confucius' by eleven, thus forming the mostly used concept in this genre. The common items are the following:

1) A bald enters a temple - to make up the number (both a bald and a monk has an egghead)

2) To borrow a comb from a monk-to baffle the person (a monk has no hair, therefore no comb)

3) A flea on a monk's head--explicit (a monk has no hair, thus a flea on his head is explicit)

4) Monks do not eat bean curd-a strange thing (monks are vegetarians)

5) A monk is under an umbrella - unruly (in Chinese hair's pronunciation is the same as rule's)

6) Monks fight against each other-no evidence for others to make good use of (hair was usually combed into pigtails in ancient China and they are easily grasped by the opponent)

7) A bachelor meets a monk - two bachelors

8) Monks divide up temple property and live apart - to do what is unnecessary (temple is similar to business in Chinese pronunciation)

'Monk' is a well-known concept in Chinese with many explicit features. An unlimited feature set of 'monk' could be depicted as this: \{monk la shaven head, reciting Buddhist scripture, a vegetarian, living in temples, a bachelor, being charitable\}. When we put these concepts into a semantic network, we can easily get the complex relationship among them when used in xiehouyu items and the feature "shaven head" is another spreading activation node for further concepts(see Figure 1):

According to the theory of knowledge graph, the ontology of word graph contains 8 dual relation groups and $4 \mathrm{n}$ relation groups ${ }^{[10]}$. In Figure 1 we sought out 3 categories of dual relation, they are: 1)SUB: demonstrating the containment of concepts in the set. 2)ALI: showing the similarity of two things. 3)PAR: presenting the attributes and intension of a concept or entity. 
In Figure 1, the nodes on the inner circle show the subordinate relations of the basic features to the word 'monk', while the concepts on the outer circle show the similarities comparing with the basic semantic concepts on the inner circle, thus a direct mapping is formed by comparing the similarities between them and the metaphors in xiehouyu items are produced by this way.

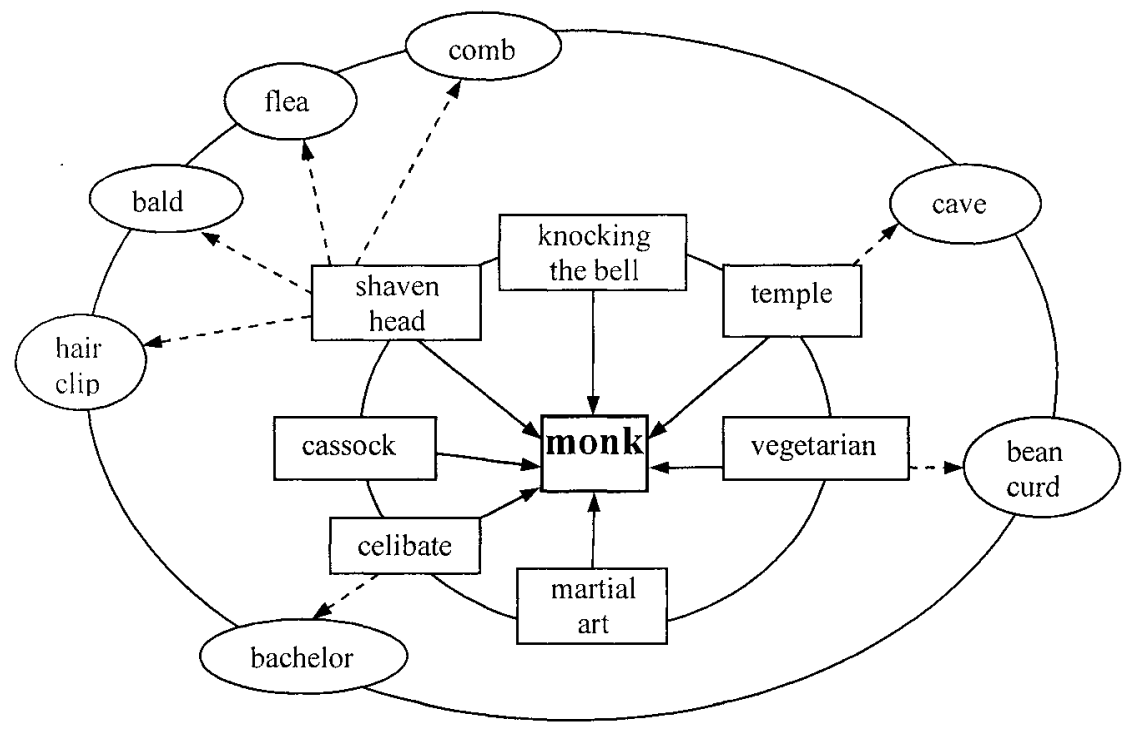

Figure 1. Semantic Network of 'Monk' in Chinese Xiehouyu Items

The core element and its activated features could be described as a generative formula according to the semantic containment and attribute of them, as the relation between the core 'monk' and features on the inner circle in Figure 1:

IF a person is a monk THEN he has a shaven head

IF a person is a monk THEN he lives in a temple

IF a person is a monk THEN he is a celibate

IF a person is a monk THEN he recites the Buddhist scripture

IF a person is a monk THEN he should do charitable things

But a generative formula would not be correct with the two parts changing their places, that is to say, the explanation of the above items would be a one-way cause and effect; hence the following formula is not appropriate or reasonable:

* IF a person has a shaven head THEN he is a monk

But if we put more than one of the subordinate concepts of 'monk' in the set together, the key concept 'monk' could be created:

IF a person has a shaven head AND lives in a temple 
AND is single

AND recites the Buddhist scripture AND ...

THEN this person is a monk.

The above generative formula demonstrates the basic elements of the word 'monk'. An important element in the feature set is the attribute element or governing element, the other important elements are subordinate element, functional element, and composing element ${ }^{[11]}$. In the xiehouyu items containing 'monk' there are no explicit features either in the riddle or in the answer because the key element of monk itself contains the basic features. In literary language the elements would not appear in the items because one feature of xiehouyu items is that they are short and concise, the usage of the basic elements would be superfluous, for example there would be no item as * 'Shaven monks fight against each other-no evidence to be grasped by the opponent'.

\section{DOUBLE MAPPING OF CHINESE RIDDLE- PUNS-XIEHOUYU}

The simile of a riddle-pun is formed by the direct mapping between the riddle and the answer, and the indirect mapping between the surface meaning and the implicate meaning of the answer creates the metaphor of this genre. The two terms of direct mapping and indirect mapping are for communication and here we borrow them for the explanation of relations among different concepts of xiehouyu items.

The expression of xiehouyu is commonly used with the second part unreleased, as the name states, when people could use general knowledge to comprehend it. This is the literary meaning of this genre. But when we take the economy principle of language into consideration, in the expressions of xiehouyu we could omit the riddles because the direct mapping exists between the riddle and the second part. But the indirect mapping between the surface meaning and the implicate meaning of the second part demonstrates the unique function of this riddle-pun and the dynamics of xiehouyu items. The indirect mapping is a means of metaphor, and it enriches people's abstract thinking. The indirect mapping establishes a joint point between the known entity and the abstract concept and let people handle new concepts with what they have known, thus a mapping across two cognitive domains is formed, just as the name of xiehouyu tells. If the first part--the riddle-was not presented, this genre would not be unique any more and the indirect mapping would not be sought out, which would not be concord with the feature of Chinese people's exaggeration of language. 
When people uses xiehouyu they just take the surface structure of the riddle as a clue for information processing, combined with background and the language context, forming a new cognition to explain the specific meaning of the riddle-pun. The semantic relation between the riddle and the answer is not isolated, but a correlated and mutually governed one.

The condition of a riddle-pun is that the riddle and the second part contain some explicit or implicit semantic relation. According to J. Oller ${ }^{[12]}$ deduction, the deduction of xiehouyu items could be explained quite clearly.

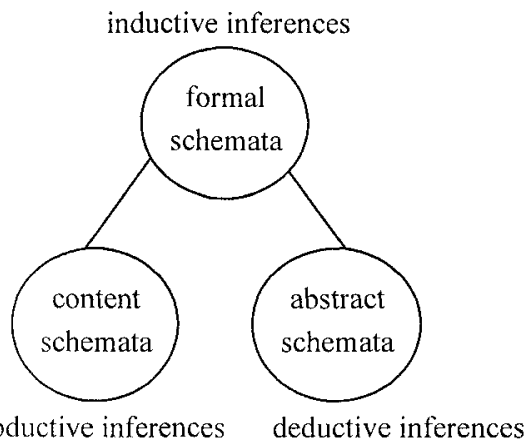

Figure 2

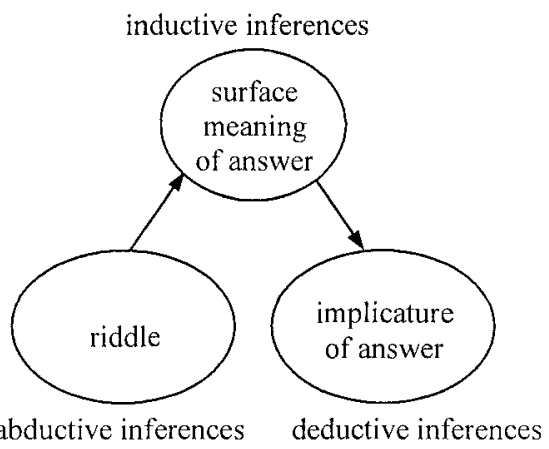

Figure 3 Xiehouyu's Deduction

Oller's three classifications of schemata are content, formal and abstract. Content schemata are defined as being based on "abductive judgments about particular facts and states of affairs". Formal schemata are the result mainly of inductive connections established across distinct states of affairs that are indexed as being similar in some respect. They seem to be developed from more distant connections of states of affairs that are somewhat similar. The third classification of abstract includes pure symbols and inductive integration. Abstract schemata carry the inductive integration to the completely general level of pure symbols. Abduction is recognizing a distinct representation; however induction is done if the recognition is more personal. The abstraction is an expansion of a single reference to act as a type through deduction. Following the classification of induction and deduction, we could easily depict the relation in the deductive direction among the three elements of xiehouyu items in Figure 3.

With deduction, a higher level of integration is achieved beyond the inductive level. Here in the item the conceptualization of the abstract concept in the answer reaches such a degree of integration and completeness that if the definition of the symbol in the riddle is sufficient, the inferences drawn from it would be equally valid and correct. 
Semantically something unusual between two domains is a premise for metaphor, and this difference is essential for the indirect mapping in the genre of xiehouyu. For example, the item 'monks fight against each otherno pigtail to be grasped by the opponent' is not only a description that monks have no pigtails for the opponent to grasp, but an extension that a person has no evidence for others to make good use of. In this item, 'monks fight against each other' is the content scheme of intuition; "no pigtail to be grasped by the opponent' is the formal scheme of deduction; and the extended 'no evidence for others to make good use of' is the highest abstract scheme of cognition.

If we take the riddle as $\mathbf{A}$, the surface meaning of the answer is $\mathbf{a}$ and it shows the relation with the riddle because $\mathbf{a}$ is the small letter of $\mathbf{A}$, and the implicate meaning of answer is $\mathbf{b}$. Therefore the deductive relation from $\mathbf{A} \rightarrow$ $\mathbf{a} \boldsymbol{\cdots} \rightarrow \mathbf{b}$ is what we usually seek out in this genre. The concept mapping of $\mathbf{b}$ deriving inderectly from $\mathbf{A}$ is implicate but attached. The relation between the riddle and the surface meaning of the answer is a simile because the hyphen could be depicted as 'be', and the relation between the surface meaning of the answer and its implicature is a metaphor, therefore this genre contains two parts of speech and this is the reason why it stands out from other styles.

Generally speaking, we could summarize four major types of mapping in xiehouyu items:

$$
\begin{aligned}
& A \rightarrow \mathbf{a} \cdots \rightarrow \text {, } \\
& A \rightarrow \mathbf{a} \cdots \rightarrow \mathbf{b}, \\
& A \rightarrow b \cdots \rightarrow b \\
& A \rightarrow b \cdots \rightarrow c
\end{aligned}
$$

All these formulae demonstrate the relation among the two concepts in the riddle and the concept of the answer in a xiehouyu item concerning the answer's weak and indirect mapping on the literal sense of the key concept in the riddle. And the formulae will be discussed in another essay.

The source category and target category of xiehouyu cognition could be built up from the relevant semantic network. In most xiehouyu items, the riddle is the source category and the answer to the riddle is the target category. In the above items containing 'monk', the key concept 'monk' does not have its trace in the answer, this phenomenon shows that that the concept of 'monk' has projected on the target category. Even though the main idea is on the second part of the riddle, it does not say that the riddle is redundant. What is the function of using xiehouyu items in some contexts? The authors think that the presence of the riddle is for the converting of surface information into the deep message-that is, the true meaning conveyed by the answer. This projection would deepen the hearer's processing effort, thus borrowing an entity to refer to the intended one is the 
rational way in Chinese literature. This cognitive process makes xiehouyu items different from other fixed expressions in Chinese.

The phenomenon of xiehouyu items could be explained from a different angle. The direct mapping between the riddle and the answer could be regarded as an example of summarizing, but not an automatic one. This is not a complete summarizing because the real intention of a xiehouyu riddle is not the truth-value of the answer. Xiehouyu items supply us good examples for summarizing because the two concepts in the riddle are used in their true meaning, though in the answer the extended or prolonged semantic concepts are projected.

Hence the riddle is a combination of two concepts, among them the word 'monk' has decreased to be a referent and the answer is the extension of the key concept 'monk' and it goes beyond the surface meaning of the words. We want to find out how close the relation between the key concept and the secondary concept in the riddle should be for the activation of relevant knowledge, or how close the riddle and the answer are for the information receiver to create a new abstract concept in their mind. Of course it needs much effort for us to find out.

So we could safely say that the usage of the riddle in expressing our mind is not to show off the literacy of ancient Chinese, it provides the hearer relevant information to comprehend the true intention of the riddle, by this the hearer could manipulate the script to activate the required information. The concepts in the riddle are only the containers and their purpose is to create a visual image in the receiver's mind, so that he could remember the true meaning more impressively. Therefore if the information processor could not deal with the riddle and activates the relevant concepts and deduces the purpose of riddle-pun, the efficiency of xiehouyu items fails to covey the meaning in Chinese traditional way.

\section{COGNITIVE MODEL REFLECTED IN XIEHOUYU}

Metaphor is not only a way to construct a new cognitive model after the cognitive model of specific matters, it also converts the inner structure and relation of the whole cognitive model to the new source, this conversion is called the mapping from the source model onto the target mode ${ }^{[13]}$. This metaphorical cognitive model would attach relevance to different categories, and it is also the effective way to simplify a language, thus reducing the difficulty of cognitive procession.

It is generally considered that in Chinese a xiehouyu item depicts a dynamic situation and presents the aesthetic function of this genre ${ }^{[14]}$. 
Xiehouyu is mainly an oral speech, and people prefer to it because it is rich and colorful to reflect people's humorous character in their daily language. This genre demonstrates Chinese peoples' cognitive means in language, which is a treasured accumulation of Chinese wisdom and culture.

The genre of xiehouyu could reflect Chinese people's thinking in borrowing a thing for analogy and extracting similarity from a category, this genre also shows the entertaining and amusing style in Chinese language. The metaphor and analogy of xiehouyu is peerless as for the other Chinese genres. Mr. Wen Duanzheng's study shows that the first part of xiehouyuthe riddle, is secondary in semantic expression but a primary role in figure of speech, which would visualize the expression ${ }^{[15]}$.

As stated in the above, the common way of forming a xiehouyu items is that it compares the similarities with other concepts. The basic category of riddle's concepts is expanded in the answer and this expansion activates people's cognition of the relevant concepts. The expanded cognitive ability would provide base for the enrichment of the definitions of concepts, and the analogy between two different concepts would also improve people's cognitive ability. The mutual development of the two concepts contrasted promote people's cognitive ability and enrich people's cognitive models about the world, during the analogical cognition Chinese culture is accumulated with colorful figures of speech.

Yao Xiyuan ${ }^{[16]}$ believes that Chinese people have the tradition to use euphemism in saying and to talk in a roundabout way, and the reserved manner in language is an important principle in Chinese. In ancient sayings there are "to borrow a nearby thing to refer to a remote idea is an eloquent expression' and 'something unreleased in the sentences and texts is the style of eloquent speakers', these sayings could be safely quoted to explain the reasons for the popularity of the genre of xiehouyu. Most xiehouyu items are good examples to reflect the fact that the riddle is a description of the familiar, visualized and concrete things, though the answer is a referent to a more abstract concept. This expression of clarifying a reserved and abstract understanding is reasonable and language economy for cognitive construal is concord with people's average cognitive models in Chinese.

\section{CONCLUSION}

We take the examples of the core element of 'monk' to analyze the semantic network and the mapping of different concepts in xiehouyu items, and we find that the riddle is not redundant but assistance to the comprehension of the true meaning of this genre. The riddles are only an onset for the information processing of it and the answers are the intended 
activated information. The answers could be regarded as results in summarizing and we can use them for reference in NLP. We hope that this genre could help us to seek out more effective cognitive models for NLP.

\section{REFERENCES}

[1] Wang Yongqing. Principles and Methods of Artificial Intelligence. Xi'an: Xi'an Jiaotong University Press. 1998

[2] Quillian M.R., "Semantic Memory", in Semantic Information Processing, M. Minsky (Ed.), MIT press, Cambridge, Mass, 1968: 227 270

[3] Song Enmin, Guo Zhiyang, Xu Zewei. A Computer System for Chinese Sentence Comprehension. Journal of Huazhong University of Science \& Technology. 1994(1) : 44-50

[4] http://www.computing.surrey.ac.uk/ai/PROFILE/prospector.html

[5] Minsky M. "A Framework for Representing Knowledge", in The Psychology of Computer Vision, P. Winston, Ed., McGraw Hill, New York, 1975: 211 277.

[6] Guo, Jianzhong. Theory and Practice of Enigmatic Folk Similes in Chinese. Chinese Translators Journal $1996(02): 12 \sim 15$

[7] Gries, P. Hays. China's New Nationalism: Pride, Politics, and Diplomacy Publisher: University of California Press. 2004

[8] The Contemporary Chinese English Dictionary (Chinese-English Edition). Beijing: Foreign Language Teaching and Research Press. 2002: 2118.

[9] Zheng Guiyou. Defaming Buddhists of Chinese Riddle-puns and Buddhism in China. Journal of Yanbian University. 1994(3): 100 104

[10] Zhang Lei, Li Xueliang, Liu Xiaodong. Knowledge graph analysis of logic words in natural language processing. Journal of Chinese Information Processing. Vol.15 No. 6: $53-58$

111) Zhang Mingming. On the Role of Semanteme and Sememe in Semantic Understandings. Journal of Northeastern University (Social Science). 2003(5) :385 387

[12] Oller, J.W. (1995). Adding abstract to formal and content schemata: Results of recent work in Peircean semiotics. Applied Linguistics, 16(3): 273 306.

[13] Zhao Yanfang. Survey of Cognitive Linguistics. Shanghai: Shanghai Foreign Languages Press. 2000: 105

[14] Li Xia. Analysis of Semantic Function in Proverbs and Riddle-puns, Forum of Folk Literature, 1998(3) : 52 54

[15] Li shuyan. Review of Study of Dialects and Vulgarism, Journal of Chinese Study, 2004(1): 56 59

[16] Yao Xiyuan. On Idiomatic Phrases. Journal of Hebei University. 1994(3): 85 91 